\title{
Das Portrait
}

\section{Bayerisches Institut für Abfallforschung (BIfA $\mathrm{GmbH}$ )}

Am 20. Juni 1991 wurde in Augsburg das BIfA gegründet, als erstes anwendungsorientiertes Forschungsinstitut in Schwaben. Es ist Bestandteil des Bayerischen Abfallforschungskonzeptes. Das BIfA orientiert sich an praxisbezogenen Abfallproblemen und ist eine unabhängige, außeruniversitäre Forschungseinrichtung, deren Hauptaufgaben wissenschaftlicher und technischer Natur sind. Zum Aufgabenbereich gehört auch die verbesserte Information von Behörden, Wirtschaft und Öffentlichkeit. Lösungen von Abfallproblemen werden nicht nur für Bayern und Deutschland erarbeitet, sondern es wird ein EG-weiter und globaler Erfahrungsaustausch angestrebt.

Seit August dieses Jahres ist die UWSF auch das Organ des BIfA, d.h. sie berichtet über dessen Aktivitäten, soweit sie für unseren Leserkreis von Interesse sind (UWSF 4/3, S. 131, 1992).

\section{Organisation}

Der Aufsichtsrat, dem Wirtschafts-Staatssekretär A. ZELLER vorsteht, trifft die Projektentscheidungen, wobei der Wissenschaftliche Beirat fachliche Empfehlungen ausspricht $(\rightarrow$ Organigramm $)$.

\section{Forschungsfelder}

1. Naturwissenschaftlich-technische Forschung

(1)Entwicklung und Verbesserung von Behandlungs-, Umwandlungs- und Verwertungstechnologien

- mechanisch

- Stofftrennung, evtl. Schadstoffentfrachtung

- physikalisch/chemisch

- Stofftrennung, Stoffumwandlung, Schadstoffentfrachtung - thermisch

- Inertisierung, Hygienisierung, energetische Verwertung

- biologisch

- Stoffumwandlung
(2) Optimierung von Produkten und Verfahren unter abfallwirtschaftlichen Gesichtspunkten

- Einsatz umweltfreundlicher Ausgangsmaterialien, Minimierung der Reststoffproblematik,

Minimierung des Schadstoffausstoßes in Produktion/Verwendung

- Verbrennungsversuche,

Überprüfung des Produktionsprozesses,

Emissionsmessungen im Betrieb und während der Verbrennung

(3) Kombination der Verfahren

Ziel: Entwicklung von Verfahrensketten mit wenig kritischen Reststoffen, geringen Abfallmengen, hohen Sekundärrohstoffqualitäten, Erreichen der Deponiefähigkeit, Hygienisierung, Detoxifizierung

Die Anwendung der Verfahren erfolgt auf

- Rückstände aus Verwertungs- und Entsorgungseinrichtungen

- Klärschlamm

- Hausmüll und hausmüllähnliche Gewerbeabfälle

- sonstige Reststoffe und Abfälle

(Sondermüll ist kein ausdrücklicher Schwerpunkt)

2. Ökonomisch-ökologische Forschung

(1)Industrielles Rückstandsmanagement

- konstitutive Merkmale von Reststoffmärkten

- Umweltverträglichkeitsuntersuchungen für Sekundärprodukte

- Ökobilanzen/Produktlinienanalysen

(2) Marktwirtschaftliche Instrumente in der Abfallwirtschaft

- Gebührenmodelle für Entsorger

- Analyse der Kostenstrukturen von Entsorgungseinrichtungen

3. Wissenschaftlich-technische Öffentlichkeitsarbeit (1)Ziele

- Verbesserung des Informationsflusses zwischen den Forschungsinstitutionen und den mit der praktischen Abfallentsorgung beschäftigten Kommunen und Unternehmen

- Informierung interessierter Bevölkerungskreise durch aktive Informations- und Aufklärungsarbeit

- Aufbereitung wissenschaftlicher Untersuchungsergebnisse und Wissensvermittlung in verständlicher und transparenter Form für Forschung, Wirtschaft und Behörden

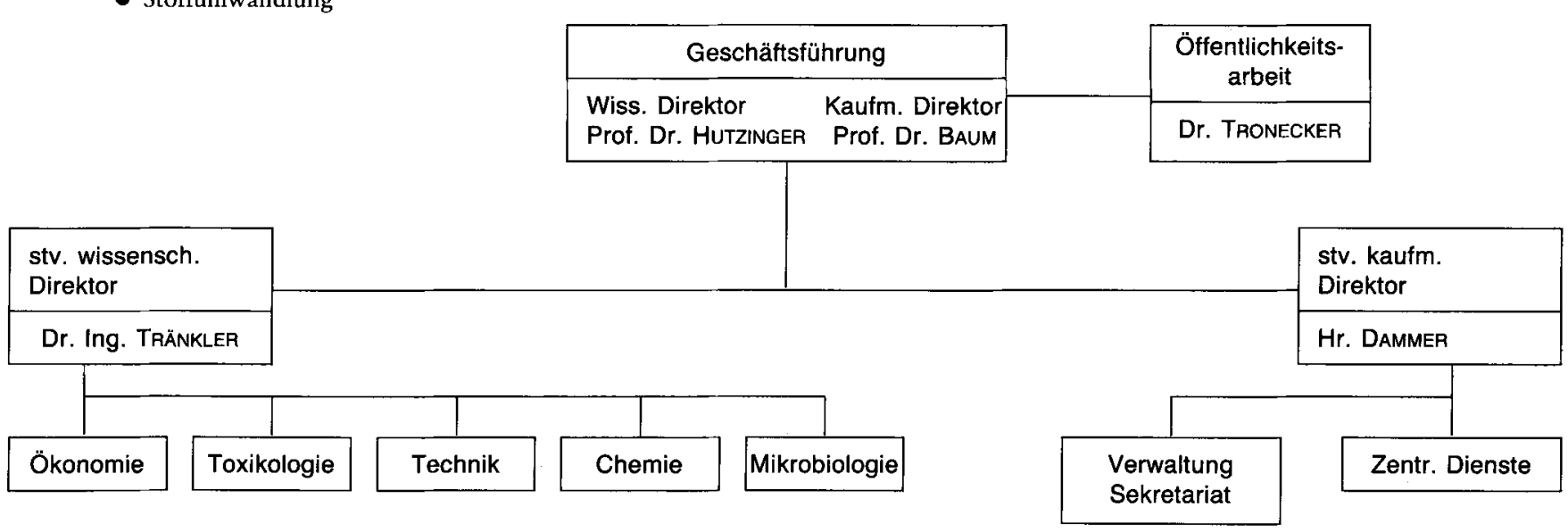


(2) Maßnahmen

PR nach außen (Öffentlichkeit und Fachkreise)

- Aktive Pressearbeit

- Ansprechpartner für die Medien

- Erstellung von Faltblättern, Broschüren und Newsletters

- Wissenschaftsjournalismus

- Verbreitung neuester F- und E-Ergebnisse

- Beratung von Behörden und Privatpersonen

- Fachzeitschrift „Umweltwissenschaften und SchadstoffForschung" als Organ der BIfA GmbH

- Zielgruppenadäquate Vorträge im Rahmen von Messen, Seminaren und Konferenzen

- Entwicklung eines Zentrums zur Aus- und Weiterbildung

- Kontaktpflege und Erfahrungsaustausch mit wissenschaftlichen Institutionen

- Zusammenarbeit mit Technologietransferstellen in Bayern

PR nach innen (Interne Kommunikation)

- BIfA Presseschau

- Interne Information

4. Aufgaben im Rahmen der staatlichen Systemsteuerung (nicht: Überwachungsaufgaben beim Vollzug von Umweltvorschriften)

(1) Betreuung von Gemeinschaftsprojekten mit Hochschulen

- fachliche Stellungnahme zu Gesetzesvorlagen im abfallwirtschaftlichen Bereich

(2) Koordination der Bayerischen Abfallforschung

- fachliche Begleitung von abfallwirtschaftlichen Pilotprojekten

(3) Wirkungsanalysen für staatliche Maßnahmen im abfallwirtschaftlichen Bereich

- wissenschaftliche Begleitung von interdisziplinären Großprojekten

\section{Forschungsprojekte}

Im Rahmen eines Statusseminars am 22. September 1992 wurden die derzeit laufenden Forschungsprojekte vorgestellt.

\section{Einfluß von Anreizsystemen auf den Erfassungsgrad von Sekundärrohstoffen}

\section{Projektbetreuung: R. WITTMANN}

Ausgangspunkt des Projekts ist der sich vollziehende Paradigmawechsel in der Abfallwirtschaft von der Beseitigung hin zur umfassenden Kreislaufwirtschaft, wie sie Gegenstand des Entwurfs zur Novellierung des derzeitigen Abfallgesetzes ist. Hinsichtlich der Strategien Vermeiden, Verwerten und Beseitigen werden die betriebswirtschaftlichen Anreize untersucht, die sich aus dem abfallgesetzlichen Rahmen für das betriebliche Rückstandsmanagement ergeben. Auf der Basis eines entscheidungsorientierten Ansatzes werden rückstandsbezogene Gestaltungsmöglichkeiten auf allen Stufen der betrieblichen Wertschöpfung (Forschung, Beschaffung, Fertigung, Vertrieb, Entsorgung) analysiert. Neben den ordnungsrechtlichen Instrumenten der Abfallpolitik (Erweiterung der Produktverantwortung durch Maßnahmen des $\mathbb{S} 14$ AbfG) stehen auch die Auswirkungen marktwirtschaftlicher Instrumente zur Internalisierung externer Kosten der Produktion und des Konsums (Abfallabgaben) im Mittelpunkt der Betrachtungen. - Der theoretische Ansatz wird durch eine empirische Untersuchung ergänzt.

\section{Kostenstrukturanalyse in der Abfallwirtschaft}

(Abt. Ökonomie im BIfA)

Projektbetreuung: J. CANTNER

Ziel dieses Projektes ist die Erforschung von Wirkungspotentialen zur Erreichung einer Trendwende vom Gemeinlast- zum Verursacherprinzip im finanziellen Bereich der Abfallwirtschaft.

Stellt man den Kosten der Abfallentsorgung das entsprechende Gebührenaufkommen der entsorgungspflichtigen Gebietskörperschaften gegenüber, wird das Defizit ersichtlich, das derzeit gemäß dem Gemeinlastprinzip von den öffentlichen Haushalten finanziert wird. Für die Behebung dieses Defizits bieten sich zwei Möglichkeiten an: Es könnten 1. die Kosten-, 2. die Gebührenpositionen angepaßt werden. Entsprechend werden die Forschungsbemühungen in diesem Projekt einerseits an den Kostenstrukturen, andererseits an den Möglichkeiten der Kostenkalkulation zur Gebührenbedarfsbestimmung ansetzen.

\section{Biomüllkompost als Dünger im Landbau - Eignung und Umweltverträglichkeit}

\section{Projektbetreuung: R. VANDRÉ}

Ziel des Forschungsprojektes ist es, die Dynamik von Nährund Schadstoffen bei Anwendung von Biomüllkompost im Landbau zu kennzeichnen. Dazu sollen in einem dreijährigen Feldversuch die Flüsse von Nährstoffen und ausgewählten organischen wie anorganischen Schadstoffen nach Kompostgaben gegen eine NPK-gedüngte Kontrolle gemessen werden. Die Erfassung von Ertrag sowie Mineral- und Schadstoffgehalten der angebauten Feldfrüchte soll eine Beurteilung der Auswirkung des Kompostes auf Pflanzenernährung und Schadstofftransfer Boden-Pflanze ermöglichen.

\section{Anaerobe Vergärung}

Projektbetreuung: H.-C. WAGNER

Gegenstand dieses Forschungsprojektes ist das Verhalten ausgewählter Umweltschadstoffe bei der anaeroben Verwertung biogener Abfallfraktionen. Als Untersuchungsgegenstand sind dabei von Interesse:

1. Akkumulations- und Metabolisierungsprozesse der Umweltschadstoffe,

2. Verteilung der Schadstoffe bzw. deren Metabolisierungsprodukte in den Verwertungsprodukten „Anaerob-Kompost“, Biogas und Abwasser.

\section{5. Ökobilanzen und Produktlinienanalysen}

(Abt. Technik im BIfA; Leitung: Dr.-Ing. W. ROMMEL) Projektbetreuung: E. WÜRDINGER

Das Aufgabengebiet umfaßt Verbesserungen der Methodik von Ökobilanzen und Produktlinienanalysen. Im Mittelpunkt stehen dabei die verschiedenen Formen der Bewertung. Ziel ist es, in Zusammenarbeit mit Unternehmen praxisgerechte Vorgehensweisen zu entwickeln, die Reichweite dieser neuen Instrumente zu bestimmen und damit sinnvolle Anwendungsbereiche abzugrenzen. 
6. Belastung der Umwelt durch atmosphärischen Eintrag vom Emissionen aus einer Müllverbrennungsanlage

Projektbetreuung: G. DÖRR, M. HIPPELEIN, H. KAUPP

Die Zielsetzung des Forschungsprojektes umfaßt fünf Punkte:

1. Erfassung der atmosphärischen Grundbelastung am Standort der im Bau befindlichen Abfallverbrennungsanlage in Augsburg;

2. Einfluß der Wettersituation (Jahreszeiten) auf Durchschnittsbelastung durch kontinuierliche Probenahme;

3. Vergleich von Meßdaten mit Ausbreitungsrechnung nach TA Luft;

4. Muster-/Profilvergleich innerhalb/zwischen den Schadstoffklassen zur Quellenzuordnung;

5. Vergleich von städtischer und Hintergrundbelastung.

Das Versuchsprogramm sieht folgende Arbeiten vor:

1. Aufbau eines Immissionsmeßnetzes: 7 Meßnetzpunkte im theoretischen Einflußbereich der AVA (Umkreis mit 2,5 km Radius); 1 Meßnetzpunkt als background im NW von Augsburg; Betrieb kontinuierlich über ein Jahr; getrennte Erfassung der Konzentrationen von PCDD/F, PCB, PAH, Hexachlorbenzol und polychlorierten Naphthalinen an Partikeln und in der Gasphase mit sechswöchiger Auflösung; Schwermetallbestimmung an Partikeln mit zweiwöchiger Auflösung.

2. Untersuchung von Kuhmilchproben auf PCDD/F, PCB, $\mathrm{HCB}$ von drei landwirtschaftlichen Betrieben im Belastungsgebiet und einem Betrieb in der Hintergrundregion; je 4 Proben Herdensammelmilch im Jahr.

3. Betrieb von je zwei Windschreibern und Thermohygrographen sowie Auswertung der Daten der DWD-Station am Flughafen Augsburg.

In einem weiteren Forschungsvorhaben soll nach Inbetriebnahme der Abfallverbrennungsanlage die Luftbelastung nochmals festgestellt und der zusätzliche Beitrag der Emissionen dieser Anlage bewertet werden.

\section{Bioabbaubare Verpackungsmaterialien}

(Abt. Mikrobiologie im BIfA; Leitung: Prof. Dr. P. WALLNÖFER, Landesanstalt für Ernährung, München, und TU München)

Projektbetreuung: P. WALLNÖFER

Biologisch abbaubare Verpackungsmaterialien werden künftig immer mehr im Vordergrund stehen, da sie das bisherige Ausmaß der Plastik-Abfälle reduzieren können. Größte Bedeutung unter den zur Zeit kommerziell angebotenen bioabbaubaren Polymeren kommt vor allem den Materialien zu, in deren Produktion die Nutzung nachwachsender Rohstoffe einbezogen wird.

Den Hauptanteil stellen derzeit Polymer-Produkte auf Stärkebasis dar, wobei Materialien aus plastisch verformbarer Stärke sowie Mischungen aus Stärke mit synthetischen Polymeren, z.B. Polyethylen oder Polyvinylalkohol, angeboten werden.
Unter den nachwachsenden Rohstoffen spielen neben Stärke auch Verpackungsmaterialien auf Zellulose-Basis eine Rolle. Dabei sind plastisch verformbare Zellulosederivate von besonderem Interesse. Die Zellulose ist hier mit Essigsäure, aber auch mit Propion- oder Buttersäure verestert und kann in Mischungen mit Polyethylen vorliegen.

Es ist nun zu untersuchen, in welchen Zeitabläufen der biologische Abbau dieser Stoffe in kommunalen Mülkompostierungsanlagen und bei der Eigenkompostierung ablaufen. Eine umfangreiche Literaturrecherche soll abklären, ob Verpackungsmaterialien auf Stärkebasis beschleunigt bioabbaubar sind, wenn der Anteil an Stärke hoch ist. Der mikrobiologische Abbau der Mischungen aus Stärke und Polyethylen ist bis jetzt umstritten. Über den Abbau der auf Zellulose-Ester basierenden Stoffe ist dagegen vergleichsweise noch wenig bekannt. Modelluntersuchungen zur Bioabbaubarkeit ausgewählter Verpackungsmaterialien auf Zellulosebasis mit speziell adaptierten Mikroorganismen sollen helfen, offen Fragen zu klären. Dabei erscheint es auch sinnvoll, mit zellfreien Systemen zu arbeiten, um mögliche Zwischenprodukte des Abbaus erfassen und bilanzieren zu können.

\section{Arbeitsschwerpunkte der Abt. Mikrobiologie}

1. Hygienische Aspekte bei Verfahren zur biologischen Abfallbehandlung

2. Überprüfung der Eignung bestimmter Abfallfraktionen für biologische Abfallbehandlungsverfahren

3. Mikrobielle Vorgänge in abgelagerten Abfallfraktionen (herkömmliche und zukünftige Deponien)

4. Optimierung von Verfahren zur biologischen Abfallbehandlung.

\section{Sachstand „Anlagen zur biologischen Abfallverwer- tung in Bayern“"}

(Abt. Mikrobiologie und Abt. Toxikologie im BIfA) Projektbetreuung: K. HOPPENHEIDT, H.-J. SCHEIBEL

Die 96 entsorgungspflichtigen Körperschaften Bayerns wurden Mitte 1992 per Fragebogen um Auskunft über die in ihrem Zuständigkeitsbereich betriebenen Anlagen zur biologischen Abfallverwertung gebeten. Eine erste Auswertung der Antwortschreiben (Rücklaufquote: $93 \%$ ) läßt erkennen, daß im Zuständigkeitsbereich der angesprochenen Behörden z.Zt. 203 Anlagen zur Kompostierung organischer Abfallfraktionen betrieben werden. $72 \%$ der Kompostieranlagen verarbeiten ausschließlich Grüngut, $23 \%$ Grüngut und Bioabfall und nur 1,5 \% ausschließlich Bioabfall. Derzeit werden nur 2 Anlagen zur anaeroben Abfallbehandlung betrieben, wobei es sich bei einer Anlage nur um eine Versuchsanlage handelt.

Die z.Zt. genutzten Anlagen zur biologischen Abfallverwertung haben $\mathrm{zu} 34,5 \%$ einen privaten, zu $33 \%$ einen kommunalen und zu $26 \%$ sonstige Betreiber (kommunal und privat,... .). Bis 1994 sollen 33 geplante Neuanlagen in Betrieb gehen, wobei es sich nahezu ausschließlich um Kompostieranlagen handelt. 
Arbeitsschwerpunkte der Abt. Toxikologie

(Leitung: Prof. Dr. W. MüCKE, TU München)

1. Risikobetrachtung zu den stofflichen Emissionen aus Abfallverbrennungsanlagen und Deponien (Toxikologie/Ökotoxikologie) unter Berücksichtigung von Gesamtbelastungen.

2. Toxikologie Kriterien für die Schadstoff-Entfrachtung von Hausmüll unter Berücksichtigung der BeseitigungsEndpunkte.

3. Etablierung mikrobieller Testverfahren zur Erfassung toxikologischer Summenparameter bei komplexen Stoffgemischen (Abfall, Emissionen) in Ergänzung bzw. als Alternative zur chemisch-physikalischen Einzelstoffbestimmung, Schwerpunkte sollen sein: Zelltoxizität, Gentoxizität und die Erfassung der Wirkung von Dioxinen und Furanen.

4. Prüfung der Eignung von Humanmonitoring zur Umgebungsüberwachung.

9. Juristisches Forschungsvorhaben

(Leitung: Prof. Dr. R. SCHMIDT)

Projektbetreuung: M. DEIKE, C. THOMA, P. NIEDERMEYER

Die Akzeptanz von Abfallentsorgungsanlagen

- Strategien zu ihrer Verbesserung

Das Projekt sucht die Ursachen der Akzeptanzkrisen zu erforschen. Es ist im Grenzbereich zwischen Rechtswissenschaft und anderen Gesellschaftswissenschaften angesiedelt. Entsprechend interdisziplinär ist die personelle Besetzung. Außerdem ist die Zusammenarbeit mit im Abfallrecht besonders erfahrenen Rechtsanwälten (Dres. HOLTWICKMAINZER, BIRKL) institutionalisiert, um den erforderlichen Praxisbezug herzustellen.

\section{Lösungsansätze}

Der spezifisch juristisch geprägte Teilbereich des Projekts beschäftigt sich vor allem mit dem Planungsrecht. Leitthese ist, daß Handhabung und rechtliche Ausgestaltung des gegenwärtigen Planungsrechts für Akzeptanzdefizite zumindest mitverantwortlich sind.

Das heutige Planungsrecht zeichnet sich durch seine geringe inhaltliche Programmierung aus. Der Verwaltung werden weite Ermessensspielräume eingeräumt, die für die Akzeptanzkrise mitverantwortlich sind, jedoch für Akzeptanzstrategien nutzbar gemacht werden könnten. Dabei sind zwei Grundfragen wesentlich:

1. die Standortfrage,

2. die Gestaltung der Anlage

(1) Bei der Standortfrage geht es darum, flächendeckend Standorte nach einheitlichen Kriterien zu suchen. In der Praxis werden die Standorte von den Entsorgungspflichtigen (den Landkreisen oder Zweckverbänden) gesucht, die sich dazu privater Unternehmen bedienen. Die Planfeststellungsbehörden prüfen dann nur noch, ob sich keine Alternativstandorte aufdrängen.
Die eigentliche Standortsuche und die Kriterien, nach denen die Standorte gesucht werden, sollten in den staatlichen Bereich zurückverlagert werden. Indessen ist für eine solche staatliche Standortsuche das geltende Recht unzureichend gerüstet:

Zwar gibt es Abfallentsorgungspläne, in denen mögliche Standorte ausgewiesen werden können; in der Praxis werden in Bayern aber nur bereits planfestgestellte Standorte oder bloße Standorträume ausgewiesen. Die Ursache für dieses Defizit liegt in dem unflexiblen Aufstellungsverfahren und in der Furcht, frühzeitig unpopuläre Entscheidungen zu treffen, insbesondere, wenn die Verbindlichkeit des ausgewiesenen Standortes nicht hinreichend gesichert ist. Es muß also darum gehen, das geltende Recht im Hinblick auf ein kriterienunterstütztes Standortsuchverfahren umzubauen.

(2) Der zweite Schwerpunkt der Arbeit bezieht sich auf die Gestaltung der Abfallentsorgungsanlagen. Ist der Standort einer Abfallentsorgungsanlage einmal verbindlich gefunden, muß es im Nahbereich der Anlage zu einer möglichst partizipativen Detailplanung kommen. Dazu bietet sich das in der amerikanischen Praxis gebräuchliche Instrument der Mediation an. Dieses Konzept versucht den Konflikt um die Abfallentsorgungsanlage auf die eigentlich streitenden Parteien, d.h. auf den Projektträger und die Anwohner, zurückzuverlagern und damit staatliche Behörden zu entlasten.

Inhaltlich sind die Ausgestaltung der Anlage, z.B. Art und Menge der zu behandelnden Abfälle, technische Ausgestaltung sowie Entschädigungsleistungen verhandelbar.

Vertrauen und Akzeptanz können aber auch durch verstärkte Kontrollmöglichkeiten (z,B. eigener Anspruch der Bürger auf Nachrüstung auf den Stand der Technik) herbeigeführt werden. Die Verhandlungslösung steht dabei allerdings immer unter dem Vorbehalt, daß die Behörde, deren Kompetenz für den Planfeststellungsbeschluß nicht beschnitten werden soll, den gefundenen Kompromiß akzeptiert. Überhaupt soll das Planfeststellungsverfahren in seiner derzeitigen Gestalt nicht angetastet werden. Es geht nur darum, bisher schon praktizierte, informelle Verfahrensweisen, dem multipolaren Charakter des Konfliktes anzupassen.

Eine solche planungsbeeinflussende Beteiligung der in ihren Rechten betroffenen Bürger setzt eine frühzeitige Beteiligung voraus. Der Zeitpunkt nach dem Scoping erscheint angemessen.

Bayerisches Institut für Abfallforschung

(BIfA $\mathrm{GmbH}$ )

Am Mittleren Moos $46 b$

D-8900 Augsburg

Tel.: 0821/27083-0

Fax: $\quad-10$ 